\title{
Thomas Blanke Streikminimierung durch Risikomaximierung - Die neue Arbeitskampfrechtsprechung des BAG
}

\author{
- Hast Di MINIMAX im Haus, \\ briche so leubl kem leuer aus"
}

Das Bundesarbeitsgericht hat in einer Serie von Entscheidungen aus der jüngsten Zeir die rechtlichen Konturen des Kollektiven Arbeitsrechts grundlcgend neu bestimmt. Auch wenn die Tragweite dicses Bruchs mit der bisherigen Rechtsprechung verbal kaschiert wird und die Grenzverläute zwischen rechemäßigen und rechtswidrigen Arbeitskämpfen insbesondere bexüglich der Aussperrung noch niche überall präzis bestimmbar sind, so zeichner sich doch ab, daß die neuerlich gewandelte Arbcitskampfkonzeption an juriscische Traditionslinien anknüpft, die überwunden schienen: Sie rehabilitiert ejne formal-paritätische Betrachtungsweise, die Streik und Aussperrung als gleichwertige und gleicheffekrive Kampfmitrel behandelt und es weitgehend der Autonomie der Koalitionen überläßr, ob, wann und in welchem Umfang sie von ihnen Gebrauch machen. Das scheinc liberal und gegenüber der judikativen Aufsich über den Einsazz der Koalitionsmittel ein begrüßenswerter Wandel.

Doch dem ist nicht so. Nicht nur, weil bekanntlich die juristische Gleichsetzung von Arbeit und Kapital in der Art und Weise ihrer kollektiven Interessenverfolgung von den strukturcllen Platzvorteilen abstrahiert, die im Konfliks um Löhne und Arbcisbcdingungen ohnedies zugunsten der Kapitalscite bestchen. Bcdenkt man, daß sich diese Platzvorreile angesichts der anhaltend hohen Massenarbeitslosigkeir, der tiefgreifenden Rationalisierungseffekte im Zugc des rcchnologischen Wandels und der ncuartigen Formen der Unternehmenskooperation noch zusätzlich crheblich verstärkt haben, so erscheint die sich abzcichnende Kehre der Arbeitskamplrechrsprechung des BAG in einem gänzlich anderen Lichr. Dies wird insbesondere darin deurlich, wie das Gericht die Risiken eincr rechtswidrigen Arbeitskampfführung und -teilnahme neu verteilc (vgl. hierzu unter II).

Bei juristischen Argumentationen weiß man selten, was sich ihre Urheber neigentlich" dabei gedache haben und worauf sie hinaus wollen. Wenn werung, Konstruktion und Argument ( $(J$. Esser) auseinanderfallen, dann bleibt das soziale Ordnungsmodell, der Kontext, in dem aus der Sicht der Richter eine Entscheidung ihren spezifischen Placz erhäle, typischerweise verborgen. Soziologische Analysen über die Gesellschaftsbilder von Richtern an den Obergerichen sind, soweir sie vorliegen, veraltet. Schon gar nicht ist untersucht und bekannt, was sich die für die Rechesprechung zum Arbeitskampfrecht verantwortlichen Richter des I. Senats des BAG untcr Gesellschaft im allgemeinen und dem Verhälınis von Arbeit und Kapira! im besonderen vorstellen. Sicher ist angesichts der normativen Unterbescimmcheit des kollekriven Arbeitsrechts, welches in der herrschenden Dokcrin wenig mehr als die allgemeine Weltohilosophie, hervorgezaubert aus und dargestellt an Art.g Abs. 3 GG ist, jedoch, daß diese Vorsicllungen - und niche etrva neuere, gar "bessere" juriscisch-dogmarische "Finsichten " - die neuerliche Wcnde im Arbcitskampfrecht geprägt haben. 
Es gilt zwar als Tugend, zu schweigen, worüber man nichts weiß. Würde diese Devise jcodoch konsequent beherzigt, so wärc alle Wissenschaft am Ende, und ansonsten würde sich gähnende Langweile ausbreiten. Daher soll eingangs kräftig spckuliert werden: über das postmoderne Gesellschajesbild - nicht etwa nur - des Hohen Senats und die Rolle, die in ihm dem Verhältnis von Arbeit und Kapital und dem Arbeitskampfrecht zukommt.

\section{Die Überflußgesellschaft. Ein postkapitalistisches Märchendesign}

\section{Der Untergang des Kapitalismus}

Irgendwann gegen Ende der 6oer. Anlang der 70er Jahre dieses Jahrhunderts ist der Kapitalismus ale und gebrechlich geworden. Schon lange Zeiten vorher wvar er gezähmt und in einen nützlichen Diener der Menschheit verwandelt worden, der wie von unsichtbarer Hand für einc ungeahnte Vermchrung der Güter und wachsenden Wohlstand gesorgt hatte. Zwar waren einige ein wenig wohlhabender als andere geworden (obwohl jedenfalls in unserem Lande alle am Anfang, als der Reichtum noch in weiter Ferne lag, mit der gleichen knappen Barschaft ausgestatter waren), aber dicse Unterschiede fielen kaum ins Gewicht, war doch soviel vorhanden, daß für alle mehr als genug da war. In die Jahre gekommen, waren dem Kapitalismus jetzt Hungcr, Armut und Elend nur mchr vage Erinnerungen an längst vergangene Zeiten.

Was die Menschen bedrängte, war nicht länger die Linderung von Not, sondern das Gegenteil: Dic Beseitigung und Verteilung des Überflusses. Kohlchalden verstellcen die Landschaft, bergehoch türmten sich die Butter-, Äpfel-, Weizen- und Fleischberge neben dem Weinsce. Soviel konnten die Leute weder essen noch trinken, obwohl sie sich reichlich darum benühten. Und überall standen dic Verkäufer Schlange, um ihre Waren und Güter zu Schleuderpreisen loszuwerden. Denn da die Menschen ihre Freihcit liebren, kam dic Einführung einer allgemeinen Abnahmeund Verbrauchspflicht leider nicht in Betracht. Immer ausgeklügeltcre Mcthoden mußten daher ersonnen werden, um den schier unermeßlichen Reichtum unter die Leute zu bringen. "Eine Wirtschafta, so konstatierten aufmerksame Zcitgenossen, ^deren Problem niche mehr die Produktion, sondern der Absacz ist, ... benötigt qualifizierte Konsumenten * (Enzensberger). Neue Geschäftszweige entstanden - sie wurden sogar bald zu den Bedeutsamsten -, die nichts anderes zu tun hatten als bekanntzumacheo, wie schön und wertvoll die neuesten Produkre seien und warum sie jedermann, wolle cr nicht unglücklich werden, haben müsse. Andere waren damit beschäfrigt, die Güter möglichst rasch veralten zu lassen und ihren kostenlosen Abrransport zu organisieren. Jedes Jalur erhielten die Menschen mehr Geld, während sie immer weniger arbeiten durf́cen. Weil auch das nicht reichte, um den Überfluß zu ihnen behende genug abfließen zu lassen, wurde ein eigenes Lohn- und Gehaltsjahr aus 13, bald i 4 Monaten cingeführt, in denen natürlich niche gearbeitet werden konnte, weil das Kalenderjahr wohlweislich sich nach wie vor mir 12 Monaten begnügtc. Sodann bekamen dic Leute noch Urlaubsgeld, damit sic die Ferien nicht zu Hause verbrachten und dort womöglich heimlich weiterwerkelten. Schlicßlich gingen die meisten Verkäufer, selbst ganze Länder dazu über, den Käufern nicht nur ihre Waren, sondern auch das Geld für deren Erwerb zu geben.

So waren zu gurer letze die Jugendblütenträume des Kapicalismus - "nur wer im Wohlstand lebr, lebt angenehm * - doch noch Wirklichkeit geworden. Wegen seiner 
großen Verdienste um die Menschheir hick man ihn, der nun selbst von seinen Kricikern liebevoll "Spätkapitalismus« genannt wurde, in hohen Ehren. Und aus Dankbarkeit gewährten ihm dic Leutc von $\mathrm{dcm}$ Vielen, was sie besaßen, cin Gnadenbrot bis an das Ende seiner Tage.

Inzwischen ist er, wie wir wissen, verschieden. Er hat uns entlassen in die Konsumund Übcrflußgesellschaft, die Dienstleistungs-, Kommunikations- und Informationsgesellschaft, kurz: in die nachindustrielle, nachkapiralistische Gescllschaft. Hier erscheint alles anders als vorhcr. Was noch an Vergangenes erinnert, erveist sich bei näherem Zuschen allenfalls als Überblcibsel, abstcrbendes Relikt, meistens jedoch als Trugbild, bloßer Schein. Der früher viel beschworene und geschmähce Klassengegensatz ist ebenso verschwunden wie der Arbeiter und sein Milieu, seine Klassenlage. Die ehemals Lohnabhängigen haben sich zu umfassend micbestimmenden Gesellschaftern, Neuen Selbständigen und stolzen Arbeitsplatzbesitzern gewandelt. Dic Überflußgesellschaft kennt weder Ausbeurung noch Entfremdung. Solidarität ist eine Parole aus verklungenen Zciten, die noch nicht im Zeichen massenhafter Bedürfnisse nach individueller Selbstentfaltung standen. Mit dem Ableben des Kapitalismus verlor auch sein Gegenmodell, der Sozialismus, gar Kommunismus, Orienticrung und Profil: Die vordem bestehende Systemkonkurrenz verblaßte und löste sich schließlich mangels Alternariven völlig auf.

Frühmanchesterliche Aufbruchstimmung herrschr wieder im Lande, nur mit dem Unterschied zu damals, daß nun wirklich jede und jeder seines odcr ihres Glückes Schmied sind oder es doch sein können. Alle starten aus dem sicheren Hafen der sozialen Geborgenheit, durch deren engmaschige Neıze auch der größte Unglücksrabe nicht hindurchfallen kann. Und wer trotzdem nicht mitkomme, sollte nicht länger die Schuld bei der Gesellschaft suchen: Sie har getan, was sie tun konnte (und miche wenige meinen, mehr als genug). Die verbleibenden Restrisiken der klcinen Unterschiede sind die leczten Wahrzeichen von Individualität und Persönlichkeit, Autonomie, Freiheit und Menschenwürde.

Doch auch die nachkapitalistische Überflußgeselischaft ist nicht frei von Sorgen. $\mathrm{D}_{3}$ von immer weniger Leuren in immer kürzerer Zeir mehr und mehr und ohnehin längst zuviel Güter hergestellt werden, finden nicht alle, die tärig scin wollen, eine angemessene Beschäftigung. So leiden viele unter der Freisetzung von Arbeit. Zudem strömen immer mehr Fremde ins Land, die an dem wirtschafelichen Scgen teilhaben wollen. Und außerdem werden die natürlichen Vorräte der Erde, dic in Form von Energie und Rohstoffen, von Luft, Boden und Wasser zur Gücercrzeugung und -verteilung nörig sind, beschleunigı aufgcbraucht, und es nicht absehbar, ob auf sie verzichtet oder für sie künstlich Ersatz beschaffen werden kann. Zwar ist es dem Fortschritt der Wissenschaft zu verdanken, daß inzwischen selbsr die Nacur nachgebaut und sogar neue Pflanzen und Lebewesen hergestellt werden können. Aber noch weiß niemand, ob und wie sie sich in den ohnehin schon arg strapazierten und verschlissenen Naturkreislauf einfügen wcrden.

Nicht alic dieser Erscheinungen sind neu. Teilweise gab es sie auch schon in der früheren kapitalistischen Epoche. Jedoch berubt diese scheinbare Übereinstimmung auf gänzlich verschicdenen, ja gegensärzlichen Ursachen. Es ist gerade nicht der Mangel, die unvollscändige oder zurückgebliebene Enrwicklung, dic dic dargestellten Schwierigkeiten der Uberflußgesellschaft erzeugt, sondem ihr Reichtum, ihre Anziehungskraft und Modernität. Die wintelligenten « neuen Medien der Informarion, Kommunikation und Produktion brauchen nicht nur eine entsprechende "intclligente Gesellschaft, sondern auch und vor allem: "intelligente Menschen die sic zum beiderseitigen Nurzen bedienen, anwenden und weiterentwickeln können. Und cben daran hapert es, weil trot'z aller Investitionen in Bildung, 
Erwachsenenbildung und Weirerbildung, rrotz aller Bereitschaft zu lebenslangem Lernen nicht alle Menschen die erforderliche Klugheit besitzen. Wenn die Arbeitslosenquote derzeit bei erwa 10\% liegt, so zeigt dies, wiewcir sich diese "Intelligenzschere inzwischen geöffnet hac. Da aus technologischen wie humanitären Gründen kein Weg zurück zu den abstumpfenden Arbeitsbedingungen des frülın Kapicalismus lührr, wird die Gesellschafe noch mehr für die Bildung aller aufrvenden müssen. Ein gervisser Bodensač freilich, in der Unrerschicdlichkeic des menschlichen Wcsens begründer und darin ethisch gerechefercigt, wird ungeachter aller Anstrengungen bestehen bleiben. Und narürlich ist die Gesellschaft in der Pflicht, sie für ein Leben ohne Arbeir durch angemessene Bereiligung an ihrem Überfluß zu entschädigen.

\section{Kaputal und Arbeit in der Überflußgesellschaft}

Es versteht sich von selbst, daß sich insbesondere das Arbeirsrecht in einer solehen intelligenten Gesellschaft nicht mehr an ideologischen Mustern festmachen kann, die aus der grauen frühkapicalistischen Vorzeit und seiner überhoiten Klassenkampfvorscellung stammen. Dessen alte Dichotomien (übrigens cher manichäischem als dialcktischem Denken entstammend) von Kapital und Arbeit, Reichtum und Armut, Akkumulation und Verelendung, Herschaft und Unterdrückung, Bourgeoisie und Prolecariat mochten dazu angetan sein, die Energien der Menschen zu bündeln und zu organisieren, um die sich entfaltende kapitalistische Produkcionsweise sozial zu fesscln und zu zähmen. Aber das ist längst Geschichte. Was von ihr blieb, war eine zeitlang noch die volltönende Agitation von politischen und gewerkschaftlichen Arbeiterführern, die vorgaben, dem längst geschlossenen sozialen Frieden nichs trauen zu können. Doch die Wirklichkeit erwies sich als stärker. So kamen auch sie nicht umhin schließlich zugeben zu müssen, was ohnehin die meisten schon wußren: Daß die Menschen ihren Wohlstand in dem Maße vermehrten, wie der Kapiralismus wuchs und gedich. So ändern sic denn allmählich ihre Ziele und Programme, um ilın zu erhalten und zu unterstüzzen, damit auch weiterhin möglichst alle an seinen Segnungen reilhabcn konnten.

Und das ging eine ganzc Weilc auch sehr gut so. Nur kurzfristig, als er bercits im Sterben lag und die Angst und Ungewißheir, wie es weitergchen und die Verceilung des Überflusses geregelt werden könnte, dic Gemüter vor allem der jungen, noch unerfahrenen Gencration verwirrtc, flackerten nochmals die alten Ideologien auf. Freilich vermochten diese Irrlichter nicht, die begrabenen Gegensätze wiederzubeleben. So kam nicht mehr als cin merkwürdiger Totentanz zur Aufführung, in dern vergangene Geister beschworen und erncur rituell gebannt wurden.

Seitdem sind die Wehklagen um den Kapicalismus verstummt. Kooperation, nichc Konflikt, Individualität, nicht Kollektivität sind Auftrag und Chance der nachkapitalistischen Gesellschaft. Dic Gewerkschaften sollten, ebenso wie dies bereits die Arbeiterparteien getan haben, ihre Rolle überdenken und gänzlich ncu bestimmen. Dies erforder zunächst ein Einsehen darin, daß sic nicht melır und anderc Rechte besitzen, gar historische (!) Aufträge zu verwirklichen haben, als jeder andere Verein auch. Sie vertreten die Interessen von ohnehin Privilegierten, den Arbeitsplaczbesitzern, deren Wohlstandsmehrung zwar angesiclits des gesellschaftlichen Überflusses unverzichtbar, aber doch zum leeren Selbsizweck geworden ist. Ihr Drang zur Gkeichmacherei behindere die Entfaltung der Phantasie und Initiative der schrumpfenden Zahl derjenigen, die noch arbeiten dürfen, und bremst den Lauf der Überflußproduktion. Und zudem führen ihre Erfolge dazu, daß die Arbeitslosen dauerhaft von der Möglichkeit zu produktiver Selbseverwirklichung ausgesperrt bleiben. 
Schon zu Zeiten des beginnenden Spätkapitalismus hatte das BAG in weiser Voraussichr erkannt, daß "Arbeirskämpfe im allgemeinen unerwünschta' sind. Heute, nach dem Ende des Kapiralismus, sind sie schlicht überflüssig. Eine intelligente Gesellschaft kann sich ausrechnen, wieviel die Menschen verdienen müsscn, um die Gü̈cr- und Warenmassen abfließen lassen zu können. Dazu bedarf es nicht länger der Rhecorik des Kampfes und des Getöses von Feldschlachicn, der gewaltsamen Behinderungen und Produktionsunterbrechungen, dic für alle Beceiligten mchr Schaden als Nuczen einbringen und zudem erhebliche Kosten für die Allgcmcinheit verursachen. Der Arbeicskampl ist vermutlich eines der leczten und sicherlich das lästigste Relikt aus den archaischen Frühzeiten des Kapizalismus, in denen für die Mehrzahl Arbeit und Leben noch cxiscentiell miteinander verknüpft waren. Entsprechend scharf wurden die hierum geführten Auseinandersetzungen ausgetragen, und die Rechtsordnung mußte dem durch begrenzce Zulassung des Konflikts ein Ventil geben. Dic Voraussetzungen, unter denen eine solche Ausnahme vom Wesen der Rechtsordnung als Friedensordnung erforderlich waren, sind längst entfallen.' Das gesellschafclich irregulär gewordene Phänomen des Arbeirskampfes, bereits heure eher eine Ausnahmeerscheinung, wird den gewandelten Vcrhälcnissen ganz van sclbst zum Opfer fallen. Diesen fälligen Abschied zu beschleunigen, ist eine der vordringlichen Aufgaben im Arbeitsrecht.

Führende Industrieverbandsvertreter, fälschlicherweise noch immer als Arbeitgeber bczeicinnet, haben die Zeichen der Zeic durchaus erkannt und nach dem Vorbild der Schweiz die nintelligente "Lösung cines Produktionspaktes vorgeschlagen, dessen Fundament eir gewerkschaftlicher Verzicht auf Arbcitskämpfe sein sollte.' $\mathrm{Da}$ jedoch die bundesdeutschen Gewerkschaften, darin ganz auf Wahrung ihres Selbstverständnisses lestgelegce Traditionsvereine, sich auf dies Angebot nicht einlassen wollien, sahen sich prominente Arbeitsrechtler veranlaßt, in die Bresche zu springen und juristisch zu sekundieren: Es gelte, die Grenzen der Tarifautonomic neu zu vermessen ${ }^{4}$ und die gewcrkschaftlichc Kampifreihcit wenn schon nicht ganz aufzuheben, so doch unzer behördliche Aufsicht zu stellen.' Und vor allem, wofür sich mit der Änderung des $\$ 116$ AFG dann auch der Gesetzgeber stark machte, die Risiken des Arbeitskamples wieder verstärkt diejenigen spüren zu lassen, die ihn führen oder doch mutmaßlich an seinen Früchten partizipieren würden. ${ }^{6}$

I BAG, GS, Beschl, v. 28.1 1955, AP Nr. I zu Ari.g GG. Arbeicskampi.

$z$ Excmplarisch fur diese Argumentation ist dic Arbelt von Picker. Dic Regelung der "Arbcits- und Wirischatubedingungen* - Vereragsprinzip uder Kampipnnzip. ZfA H. j/1986. S. 199 fi.

1 Dic Richtung der Nachdenkens, wie *man intelligenter miteinander umgehe $\alpha$, gab D. Kirchner, Hauptgeschäluführer von Gisame-Mctall, auf Jer wissenschafflichen Konferenz des DGB am 17. und 18.3.1987 in Ludwighafen wic folgt an: $\times$ Mir schwebre wirklich vor. daß wir eines Tages sagen konnicn: Zukunfi ohnc Arbeikskampfe slall, Zukunfl des Arbeitskaniflesea, vgl. das Protukall in: G. Muhr (Hrsg.), Streikrecht, Demokratie und Soztalsaat, Koln $1987,5.251$ und 25$\}$.

4 Adomeit, Grenzen der Tarifautonome - neu gezogen, NJW 1984، S. 595 f. Nach ihm ist der Tarifvertrag eine "Gewinnausschüttungsverenbarunga. Wo nechts zu verteilen sst, zwngen gleichwohl vereinbarz Lohnerhöhungen oder Arbeitszestverkürzungen die Untcrnehmen in die rolen Zahlen. Das aber erwesst stch für alle Arbentnehmer als noch ungünsuger, folglich sind derantige Tarilvectrïge wegen Verstoßes gegen das Gunsugkeresprinxip nichug.

s Riuthers, Funktionswandel im Arbeisseche, ZfA H. 3/1988, 5. 257ff., pladierc anliand des gewerkschaftliehen Mibbrauchs der Tarifautonome vor allem im Öffentlichen Dienst, aber auch im Bereich von Monopolen oder Oligopolen oder in staxtich subventionieren Wirschnfiszwesgen dafü. - in den Tarıfberesclien, in denen geeignete Grenzrisiken felyen, ... ene starliche Tarifaulsich nach Art des Kartellames einzurichtena (\$. 26) ).

6 Zur Gesetzgebungsgeschıchte und -krnik vgl. Mückenberger, \$ 16 AF G: Stadien eınes Gesectzgebungsprozesses, KJ H. 1/1986, S. 166 if. und dgl. Reprivatisicrung der Arbeitskimplíolgen. KJ H. $3_{1 / 986 \text {, }}$ S. 281 if. 
In solcher Zeitenwende hatte das BAG in einer Rcihe von Enrscheidungen Gelegenheit, Zulässigkeit und Grenzen des Arbeitskamples als wichtigste und quasi gescizgebende Instanz neu zu vermessen. Worin auch immer das Gesetz der Serie begründet sein mag, im Rhythmus von ungefähr acht Jahren häutet sich seine Rcchtsprechung, und so war nach den Etappen von $1955^{7}, 1963^{\circ}, 1971^{\circ}$ und $1980^{10}$ mit einem neuerlichen Durchbruch zu rechnen. Er ist, sovicl sci vorweg angedeuter, nur zum Teil genutzt worden. Aber die Richtung, immcrhin ein Trost, stimm wenn auch auf intelligenten Umwegen.

Bereits in seiner Kommenticrung der BAG-Entscheidungen zum Arbeitskampfrecht aus dem jahr 1980 hate ein prominenter Arbeitsrechrler frohlockend bemerkt, daß dic Arbeitskamplfreiheit auf Arbeitgeberscite durch die Beschränkung der Aussperrungsbefugnis auf eine reine Defensivnaßnahme, zudem quotenmäßig regulicrr, ror sei und es nur mehr eine Frage der Zeir sein werde, bis gleiches auch für dic Arbeitnehmer- und Gewerkschaftsseitc gelten wordc." Dies schien Konsens, wurde doch auch von der entgegengeserzten Warte die Zukunft des Arbeitskamples in einem staatlich verordneren Ritual gesehen. ${ }^{\prime 2}$

Indes, dies waren ersce Reaktionen, die noch von analycischer Schärfe und diagnostischem Mut gekennzeichnet waren. Seitdem zerfiel die Debatte wieder in ihre eigentümlich unzeitgemäßen, noch dem Kapiralismus verhafteten Lagermentalizäten, wobei dic stärkcren Bacaillone sich unzweifelhaft auf Seiten der Arbeitgeber versammeiten und insbesondere die Strangulierung der Aussperrung's, die unzureichende Risikobelastung der Arbeinehmer und Gerverkschaften's sowie die Zulassung systematisch organisierter gewerkschaftlicher Warnstreiks wäbrend laufender Tarifverhandlungen's attakierten. Daß sie dabei, nach dem diletrantischen und selbst von den Gewerkschaften abgelehnten Vorstoß der Bundestagsfrakcion der „GRÜ$N N_{*}$, die Aussperrung gesetzlich zu verbieten ${ }^{16}$, sogar soweit gingen, die Forderung nach einer umfassenden geselzlichen Regelung des Arbeitskampfrechts massiv zu propagieren ${ }^{17}$ und hierzu endlich auch einen Entwurf vorzulcgen ${ }^{18}$, mußte das oberste Arbeitsgericht in Zugzwang setzen: Es gab etwas zu korrigieren, soviel war klar. Aber was? In welche Richtung? Cui bono?

7 Vgt. oben Fn. I.

8 BAG, Ure. Y. 20.12. 1963, AP Nr. 3z zu Ar., GG, Arbestskampr.

9 BAG, GS, Beschl. v. 24. 3.1971, AP Nr. 43 zu Art. 9 GG, Arbiltskampi

10 BAG v. 10.6.1980, AP Nr.64 und 65 zu Art.9 GG, Arbeiskampr.

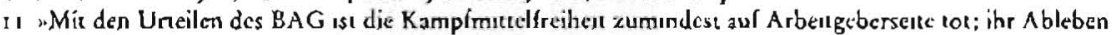
auí Arbeitnelımersere ... scheınt mir nur ane Frage der Zerr. Der Allgcmeinhelt kann dies nur rechı scin", so Lieb, Gedanken zur Aussperning, DB 1980, S. 2188 ff., z192.

I2 Mückenberger. Der Arbetskampl als staatlich inszenertes Rilual, BIStSozuArb 1980, S. 24 , if., 217 if.

13 Vgl. erwa Lieb (Anm. I I) und Setter, Die neue Aussperrungsrechtsprechung des Bundesarbeitsgerichis. RdA 1981.

$14 \mathrm{Vgl}$. etwa Isensec, Die Neuregelung der Arbescskampf-Neutralität nach \$136 AFC und dic Vorgaben der Verfassung. DB 1986, 5.429ff,; Rasser, Dic Neutralitätspflicht dor Bundesanscalt für Arbent im Arbciskampf, NZA i 984 , S. 369 ff.; Papier, Dic verfassungsrecheliche Diskussion um den nSercikparagraphen«, ZRP 1986, S. 72 Jf.; Schulin, Arbeieskampl und Neutalitar der Bundesinstale für Arbert, DB

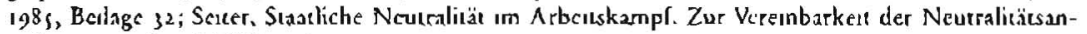
ordnung mis $\$ 116 \mathrm{AFG}, 1985$.

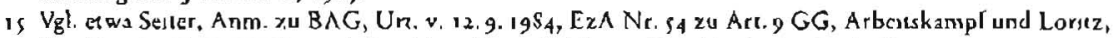

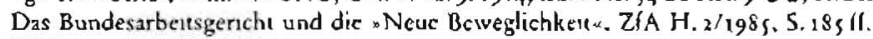

16 BT Drs $10 / 1635$ v. 18.6.1984.

$17 \mathrm{Vg}$. erwa die Betrage von Richardi, Kirchner, Sciter und Froul auf dern RdA-Symposium zum Arbeisskamplrecht, abgedruckt in RdA H. 3,4/1986, S. 141 ll.

i \& Gesetz xur Regelung kollekeiver Arbeitskonflikec, Encwurí und Begründung, Tübungen ig\$, vgl. herzu die kriusche Darstellung bea Mückenberger, in diescon Heí S. $2_{4}$ ilf. 

Risiko, weniger Kampf

Auf den ersten Blick erscheinen die jüngsten Entscheidungen des Bundesarbcitsgerichts zum Arbeicskampfrecht wie ein Flickenteppich: $\mathrm{Da}_{2}$ wird, zum wer weiß rvievielten mal, judiziert zur Aussperrung's (und ihrem Verbor in der Hessischen Verfassung), zum gewerkschafrlichen Warnstreik ${ }^{20}$, dem Sympathie- oder Solidaritärsstreik"1, dcm "wilden « Strcik*z, zur Organisations- und Aufsichtspflicht der Gewerkschatien und ihrer Organe bei der Durchführung von Streiks ${ }^{23}$, zur Schadensersarkpfliche von Streikenden, Streikleitungen und Gewerkschaften ${ }^{24}$ sowie zu Unterlassungsansprüchen der Tarifvertragsparteien auf rechtswidrigc Arbeitskampfmaßnahmen der Gegenseite. ${ }^{25}$ Und fast überall, Innovation ist Trumpf, finden sich neue Kreationen. Das Arbeitskampfrecht ist neuerlich in Bewegung geraten - doch wohin stcuer oder aber: schlingert es?

Der Versuch, ein einheitliches Muster auszumachen, wird zunächst erschwert durch die Tatsache, daß sich das Gericht erstens relativ strikt an die jewcils zur Entscheidung anstehende Frage hält und allgemein gehaltene, fallübergreifende Ausfübrungen meides, zum anderen durch die üblich re Technik der Darstellung, die bemühe ist, auch Neuerungen möglichst als Fortführung der Tradicion auszugeben. Gleichwohl ist davon auszugehen, daß der I. Senat des BAG einc durchaus konsequente Neukonzeption des Arbeitskampfrechts verfolgt, deren Konsequenzen freilich erst in Ansätzen sichtbar werden.

\section{Arbettskampl als Medium der Tarifautonomie}

Zweifellos in der Kontinuitäc seiner bisherigen Rechtsprechung steht die Hinordnung des legirimen Arbeicskampfes auf das Tarifvertragssystem, die in ihren Konsequenzen allerdings noch strikter als bisher durchgefühn wird: "Die Funkrion des Arbeitskampfes bestimm die Grenzen seiner Zulässigkeit. Der Arbeitskampl ist wegen seiner Hilfsfunktion für die Tarifautonomie nach Art. 9 Abs. 3 GG i. V. mit $\$ 2$ TVG gewährleistec und zulässig. Er ist ein Hilfsinstrument zur Sicherung der Tarifautonomie. Deshalb darf er auch nur als Instrumenc zur Durchserzung tariflicher Regelungen eingesetzt werden. Ein weicergchendes Streikrecht läßt sich Art. 9 Abs. 3 GG nicht entnchmen ${ }^{26}$ Von dicser Prämisse ausgehend bekräftig̨ das BAG abermals dic Rechtswidrigkeit von nichtorganisierten, "wilden * Streiks sowic von gewerkschaftlichen Sympathie- oder Solidaritärsstreiks"7, durch die der Arbeitskampf einer anderen Gewerkschaft um den Abschluß eines Tarifvertrages unrerstützt werden soll. Zwar betonc das Gcricht zunächsז unter Bezugnahme sut sein Urteil vom S.3. $1985^{28}$, daß Sympathiestreiks lediglich nin der Regelu rechtswidrig

19 Ur. v. $26.4 \cdot 1988, \mathrm{DB} 1988$, S. 1902 ff, und v. 7.6. $1988, \mathrm{DB} 1988, \mathrm{~S} .210_{4}$ f.

20 Ur. v. 21.6.1988, DB 1988, S. $195=$ It.

21 Urt. v, 12, 1, in8s, DB 1988, S. 1270 = AiB $5988,5.18$, lf

22 Un. v. 7.6.1988, DB 1988, S. $2102=B B 198 \%$, S. 50$\}$ f.

23 Un. v. Un. v. 21.6 .1988 (Fn. 20), Un. v. 21.6. 1988, BB 1989. S. 502 f. und Urt. v. 8.11. 1988, DB 1988 , S. 2363 (Presseinformation)

$24 \mathrm{Vgl} . \mathrm{Fn}, 2\}$.

2s Ur. v. 26. + 1988 (Fn. 19) und die in Fn. 23 angegebenen Entscheidungen. Zur Analyse und Kritik dieser Urteilskete vgl. auch H. Wolzer, Richterrecht und gewerkschaftliche Strcikfähıgkeıh, ArbuR 1988, S. 357 li:

26 Urt. v. 7.6.1988 (Fn. 22) DB 1988, S. 2102.

$27 \mathrm{Vgl}$. Fn. 21 und 22

28 BAG AP Nr. 8; zuAn. \& GG, Arberrskampt. 
sind, ausnahmswejsc also durchaus zulässig sein können. Jedoch handelt es sich hierbei, wie aus den weiteren Ausführungen hervorgeht, nur mehr um einen verbalen Vorbehalt. Denn weder ergebc sich die Zulässigkeit von Sympachiestreiks die ÖrV hatte im Arbeitskampf der IG Metall um die 35 -Stunden-Woche im Jahr 1984 zu Solidaritätsveranstaltungen während der Arbeitszeit aufgerufen - hier ausnahmsweise daraus, daß sic von vorneherein auf eincn bestimmten, kurzen Zeitraum beschränkt waren. Noch seien sie etwa dann gerechtfertigt, wenn andere Gewerkschaften kleineren, die nicht in der Lage sind, einen wirksamen Arbcitskampf um einen Tarifvertrag zu führen, zur Herstellung tarifbezogener Parităt zu Hilfe kommen. Denn erstens ist nach Auffassung des BAG »diese tarifbczogene Pariär ... nicht dadurch gestört, daß im Tarifgebiet die eine Tarifvertragsparzei sich im Einzelfall tassächlich mächtiger erweist als die andere ${ }^{29}$, und zweitens isc es „Sache der Arbeirnehmervercinigung, diese efforderliche Mächzigkeic zu erwerben und zu bewahren, wenn sie als tariffahige Koalition an der Tarifautonomie teilhaben swill $\psi_{2}$,

Wenig später, in seiner Entscheidung vom 7.6.1988 zum "wilden* Streik läßt das Gericht denn auch konsequent die Rede von Regel und Ausnahme fallen und glättet seine bisherige Judikatur: „Bercits die Zulässigkeit des Sympathiestreiks hat der Senat in ständiger Rechesprechung verneint, weil er nicht unmittelbar der Herbeiführung eines Tarifvertrages dienc. $x^{\text {)' }}$

\section{Rückkebr zur Rechtsfiktion der formellen ArbeitskampSparttät}

Legiciom ist demnach grundsätzlich und, so wird man hinzufügen müssen, ausnahmslos nur mchr der unmitcelbar um tarifvertragliche Regelungen geführre Arbeitskampf. Erkennbar wird weiterhin, daß der I. Senat des BAG dazu tendiert, das Konzept der carifbezogenen Paritäc wieder stärker formell zu fassen und es eincr materialen, empirisch-sozialen, historischen sowie rechesfunktionalen Betrachtung, aus der das Gerichr in seincn Entscheidungen vom 10.6.198031 die Beschränkung der Aussperrungsbefugnis hergeleiter hatte, zu entziehen. Auch wenn dic realen Kräfteverhältnisse unparitäcisch sind, ist dies für den arbeirsrechclichen Paritätsbegriff irrelevant. Folgerichtig rücks das Gericht in seiner Ausspcrrungsencscheidung vom 7.6. $1988^{13}$ denn auch erstmals deurlich von den Grundsätzen seiner Entscheidungen vom 10.6. 1980 ab und reilt mir: "Der Senat hat Bedenken, ob an den Maßstäben der Entscheidung yom 10.6. 1980 für die Zulässigkeit einer Abwehraussperrung festgehalten werden kann ${ }^{34}$ Bereits scinem Urteil vom 26.4.1988's, in dem es erneut die Nichrigkeit des Aussperrungsverbots dor Hessischen Verfassung wegen Verstoßes gegen die im Tarifreche des Bundes enchaltene Aussperrungsgaranrie festgestclle hatte, war zu entnehmen, daB das BAG seine Rechesprechung wieder primär an der Entscheidung des Großen Senats vom 21.5.1971 ${ }^{36}$ auszurichten gedachte. Die Ausspecrung, so hatte das BAG seine ständige Rechtsprechung zusammengefaßr, isc *als Kampfmituel zur Errcichung eines rariflich regelbaren Zicles grundsärzlich zulässig ${ }^{37}$ - diese Formulierung läßr alles offen und schließ

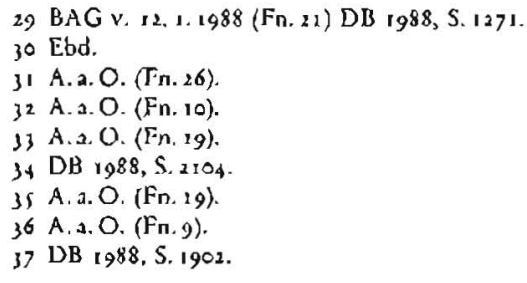


auch die Angriffsaussperrung keineswegs aus. Die Legicimationsbasis der Aussperrung verbreitert sich: In seinem Urteil vom 7.6.1988 wird die Zulässigkeir der Abwehraussperrung in Reaktion auf enggeführte gerverkschafticle Teilstreiks nun nicht mehr allein aus dem Gesichtspunkt gerechtertigt, daß durch die Gefährdung der Arbeitgebersolidarität einc Stönng der Kampfparität bewirkt werde. Vielmehr könne leiztere auch andere Ursachen habcu, die freilich niche näher dargecan werden; jedenfalls komme cine Bescluränkung der Aussperrungsbefugnis auf Unternehmen, dic zu schon bestreikten Unternchmen in Weitbewerb stehen, nicht in Betracht. ${ }^{8}$ Die Frage, wo anstelle der früheren Quotenregelungen die Grenze der zulässigen Abwchraussperrung zu ziehen sei, läßt das Gerichr unbeantwonet, stellt aber iest, daB jedenfails die Zahl der "kalt ausgcsperrten « Arbeitnehmer hierbei nicht mic zu berücksichrigen sei. ${ }^{39}$

\section{Preisgabe der ultuma-ratio-Doktrin}

Noch deutlichere Konturen erhält das neue Arbeitskampfkonzept des 1. Senats in seiner Warnstreikentscheidung vom 21.6.1988 Insider der Materie so überraschend kamen, daß bis zur Vorlage der schriftichen Urteilsgründc allgemeines Rätselraten über die Tragweitc der Veränderungen herrschte. Auch jetzr sind sie noch kaum abzuschätzen, so gravierend ist die Korrektur seiner bisherigen Rechtsprechung. ${ }^{402}$ Nach der ersten Entscheidung des BAG vom 17.12.19764' zur Zulässigkeit des kurzen, verhandlungsbegleitenden gewerkschafelichen Warnstreiks hatcen die Gerverkschaften die Taktik vorübergchonder Arbeitsniedcrlcgungen während laufender Tarifverhandlungen zur Strategie der sog. wneuen Beweglichkeitu wciterentrvickelt. Das BAG hatte auch diese Strategie im Sinne sciner Ausgangsentscheidung mit seinen Urteilen vom 12.9. $1984^{42}$ und vom 29. 1. $1985^{\text {4s }}$ für zulässig erachter, weil durch sie nur ein »milder Druck auf die Arbeitgeberseite ausgeübt worde und sie dazu gecignet seien, infolge der Demonstration der Streikbereitschaft der Arbeitnehmer die Einigungsprozesse in den Tarifverhandiungen zu beschleunigen, so daß dic Durchführung von Erzwingungsarbeitskämplen vermicden werde. Dies rechtfertige es, sie von dem ansonsten geltenden wultima-racio-Prinzip», wonach Streiks nur als letztes Mitcel der Konfliktaustragung nach dem Scheitern der Tarifverhandlungen zulässig seien, auszunchmen. Diese Argumentation hat das BAG nunmehr preisgegeben. Allerdings nich, wie vielfach gefordert (und befürchter), durch die Wiedererrichtung des Verbots verhandlungsbegkeitender Warnstreiks oder zumindest ihre zeitliche und sachliche Beschränkung (enva: einmal pro Berrieb lür maximal ein bis zwci Stunden). Vielmehr formulicrs es im Gegenteil die multima-ratio-Doktrina in der Weise um, daß der Warnstreik keine arbeitskampírechtiche Privilegierung mehr erfährt und künfrig alle ansonstcn erlaubten Arbeitskampfformen auch während laufender Tarifverhandlungen zulässig sind.

Ausgangspunkt der Entscheidung ist dic Feststellung, daß sich wangesichts der

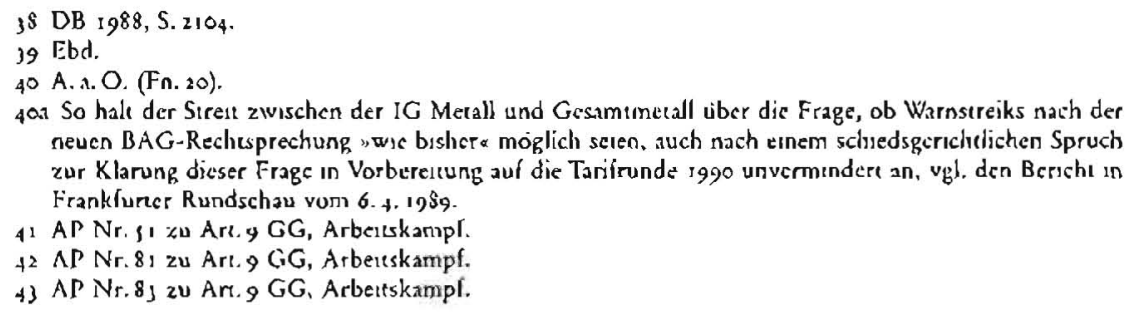


Entwicklung der Warnstreik-Praxis in den letzten Jahren ${ }^{-14}$ diese Kamplform niche mehr in rechtlich relevanter Weise von anderen Arbeitskampfformen uncerscheiden lasse. Weder Dauer und Häufigkeit noch Intensitär und Schadenshöhe bildeten verläßliche Abgrenzungskritcrion zwischen Warn- und Erzwingungsstreiks, jede nsolche Definicion ware willkürlich, sie fände in der Rechtsordnung keine Grundlage"." Aber auch die in der Literatur angeführten funktionalen Unterschiede beider Streikformen rechtfertigten nicht ihre rechtliche Ungleichbehandlung: Weder könne differenzier werden wzwischen einer Druckausübung mi dem Ziel, überhaupı oder zügig oder kompromißbereit zu verhandeln, und einer Druckausübung mit dem Ziel, zum Abschluß eincs Tarifvertrages zu kommen " ${ }^{46}$, noch sei die Demonstrationsfunktion ein zureichendes Abgrenzungsmerkmal zwischen Warnund Erzwingungsstreik, weil ersterer auch »unmitrelbar materielle Druckausübung durch die Folgen und Schäden der Arbeicsniederlegung selbst (ist) $\alpha$ und nauch der Erzwingungsstreik herkömmlicher Art nach Scheitern der Taxifverhandlungen .... jeweils gleichzeitig Kampfbcreitschaft für die Zukunft (demonstriert) und damit... der möglichst baldigen Wiederaufnahme der Tarifverhandlungen (dient) $\alpha .+7$. Schon gar nicht gerechrferrigt sci eine juristische Privilegierung des Warnstreiks durch die Erwägung, er sei als notwendiger Test der Arbeitskampfbereitschaft ihrer Mirglieder und der Außenscitcr für die Gewerkschaften unverzichtbar. Dies möge zwar so sein, jedoch dürfe ndieser Tese als innergewerkschaftlicher Vorgang ... nicht zu Lasten der Arbeitgeberscitc erfolgen.$^{.8}$ Folglich lchnt das Gericht jede Differenzierung zwischen Warn- und Erzwingungsstreiks ab: warnstreiks auch in der Form der neuen Beweglichkeit unterliegen daher dem ultima-ratio-Prinzip $\alpha .{ }^{49}$

Dieses Prinzip erhält aber einen grundlegend gewandelten Bcdeutungsgehalt. Nach der nunmehr geläutcrien Auffassung des BAG erfordert die Formel des Großen Senats vom Gebot der vorherigen "Ausschöpfung aller Verständigungsmöglichkeiten so $^{30}$ weder die einvernehmliche, eindeurige und offizielle Erklärung der Tarifvertragsparteien, daß die Verhandlungen gescheitcrt seicn. Noch könne dieser Zcitpunkt matcrial definierı werden, da dies zu unzulässiger Tarifzensur fülıre. Vielmehr sei seinc Bcstimmung Sache jeder einzelnen Tarifpartei: In der Einleitung von Arbeitskampfmaßnahmen liegr ... die freie und nicht nachprüfbare und daher allein maßgebcnde Erklärung der Tarifvertragspartei, daß sie die Verständigungsmöglichkeiten ohne Ausübung von Druck als ausgeschöpft ansieht. Es gibr damit auch keinen weiteren maßgebenden späteren Zeitpunkt, von dem ab erst andere Arbeirskampfmaßnahmen als Warnstreiks, auch solche des andercn Tarifparcners, zulässig sind. Von diesem (einheitlichen) Zeitpunkt an ist cin Warnstreik wie jede andere Arbeirskampfmaßnahme, auch während laufender Tarifvercragsverhandlungen, nicht ausgeschlossen . $^{\prime \prime}$

Damit waren in dem zu entscheidenden Fall die Warnstreiks der Gerverkschaft HBV zulässig, obwohl sie zu einem Zeilpunkt durchgeführt wurden, an dem die spätere Fortseczung der Tarifverhandlungen bereits beschlossen war - selbst während laufender Verhandlungen wären sie zulassig, wcil auch umgekehrt "Verhandlungen über den Neuabschluß von Tarifverträgen ... während der Daucr von

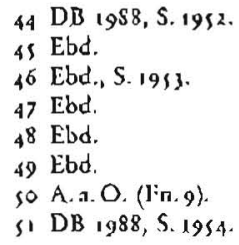


Arbeirskampfmaßnahmen üblich (sind), ohne daß daraus Folgerungen lür die Zulässigkcit der laufenden Arbeitskampfmaßnahmon gezogen würdenw.\$2 Aber, und das is der springende Punkt der Entscheidung, ibre Zulässigkeit ergibu sich nicht aus ihrem Charakter als Warnstreiks, der nwie jede andere Streikform ein Erzwingungsstreik ist "s3, sondern aus der Relativierung des ultima-rario-Prinzips, dic auch jede andere Arbeitskampfmaßnahme während laufender Tarifverrragsverhandlungen zulässig machr. Die Bedeutung dieses Prinzips als *Ausformung des Grundsatzes der Verhälenismäßigkeitu's reduziert sich demnach darauf, daß „zuvor Forderungen für den Inhalt des abzuschließenden Tarifvertrages erhoben worden sind und daß in der Regel über diese Forderungen auch Tarifvertragsverhandlungen geführt wurden; eine Ausnahme gilt dann, wenn die andere Seite Verhandlungen über cine Forderung uberhaup ablehnt «" "s

Mit der juristischen Privilegierung des Warnstreiks ist es also vorbei. Strcik ist Streik, die feinsinnigen juristischen wie sozialwissenschaftlichen Unterscheidungen der Streikformen sind irrelcvant, und sind erst einmal die Tarifverhandlungen aufgenommen, können Arbeirskampfmaßnahmen ergriffen werden. Angesichts der drohenden Tarifzensur wird man weder wemsthafte* Verhandlungen noch gar Kompromiß̧bercitschaft vor Kampfbeginn verlangen können. Und nichts spricht dafür, daß sich das Privileg des Warnstreiks nun gar zum Kampfprivileg der Gewerkschatten gemausert haben könnte: Da Warnstreiks Erzwingungsstreiks sind, kann ihnen jedenfalls mit der nichr mehr quotenmäßig begrenzten Defensivaussperrung im Rahmen der Verhälnnismäßigkeit begegnet werden.

Ob auch verhandlungsbegleitende Angriffsaussperrungen im Kalkül der neuen Arbeitskampikonzeption des BAG liegen, ist gegenwärtig nicht absehbar. Offenbar wollte sich das Gericht, wie die Begründungsstrategie des Warnstreikureils zeigt, soweit nicht festlegen. Denn andernfalls hätce es nahegelegen, den argumentaciv aufwendigen Weg der rechrlichen Planierung der diversen Streikformen und die fakrische Preisgabe des ultima-racio-Prinzips zu umgehen und kurzerhand auf dasjedenfails nach der ganz überwiegenden Doktrin - alles beherrschonde Grundpinzip des Arbeitskampfrechts zu rekurrieren: das Paritätsprinzip, welches in seiner simpien Logik des "Was dem einen recht ist, ist dem andern billig von hoher Selbstcvidenz ist und den Arbeitgebern jedenfalls das Recht zur verhandlungsbegleitenden Wamaussperrung gegeben härte.

Die von BAG gewählıe Alternative iss zudem im Hinblick aul das Ziel, Arbcirskämpfe ihrer eigenen Überflüssigkeit zu überführen, riskant: Können die Tarifgegner, nur unヶureichend gebändigt durch die vagen, erst nachträglich eingreifenden Maßstäbe der Verhälınismäßigkeit, Gecignerheit und Erforderlichkeit ihrer wechselscicigen Kampfmaßnahmen sogleich gegeneinander vorgehen, so sind Arbeitskämpfe nach Beginn und Ausmaß kaum vorhersehbar und können überfallartig ausgelöst werden. Dadurch entfällt die pazifizierende Wirkung des Faktors Zeit, der im Arbeitskampfrecht traditionell große Bedeutung beigemessen wurde und der erwa durch die Statuicrung von Verhandlungspflichten, Schlichtungsregelungen, Abkühlungsphasen und Ankündigungsfristen vor Arbeitskampfbeginn oder eben durch das uktima-ratio-Prinzip Raum zur Entfaltung gegeben wird.

Aber wer weiß, welche Envägungen das Gericht bei sciner Entscheidung gehegt hat. Im Kontext des oben unter I. ausgebreiceten Märchenszenarios mag es davon

is Ebd. 
ausgehen, daß in der nachkapicalistischen Gesellschaft desro weniger Arbeicskämpfe stattfinden, je größer auf beiden Seiten die - freilich auf tarifliche Auseinandersetzungen beschränkte - Arbcirskamplfreiheit ist. Dafür spricht, daß die Zeiten vergangen sind, in denen typischerweisc immer nur die eine Seite, dic Gewerkschafren, Forderungen an die Gegenseite nach Lohnerhöhungen, sozialer Absicherung oder Arbeitszeitverkürzung stellte. ${ }^{56}$ Inzwischen steht dagegen das Anliegen im Vordergrund, die vormodernen kulturellen und arbeitsrechdichen Blockierungen der Überflußproduktion zu bescitigen: wncelligenter Produkrion erforder die Rundumnurzung der Technologieparks, dic Gleichberechrigung aller Wochentage und dic Möglichkeit zur individuellen Ausgestaltung der Arbeitsbedingungen entsprechend den Bedürfnissen der Arbeitnehmerpersönlichkeir. Deshalb muß die Árbeitgeberseite auch die Möglichkeic crhalten, ihrcrseits dicse Modernisierungskonzepte in Tarifverhandlungen einbringen und ihnen notfalls mic Kampimaßnahmen Nachdruck verleihen zu können. Daß dabei die zeitlichen Spielräume geopfert werden, die vordem zur Vorbereitung von Abwehrmaßnahmen gegen Streiks genutzt werden konnten, und der Faktor Zeit insgesamt als Kalkulacionsmasse schwindet, ist zwangsläufig. Aber dieser Verlust war infolge der technologischen Entwicklung ohncdies nicht aufzuhalten, weil zunehmende Fcrtigungsciefe, vcränderte Produktionsmethoden und unternehmensübergreifende informationstechnologische Feinabstimmung der Produkcionskoordination, fehlende Lagerkapazitäten und Vorratshaltung dazu geführt haben, ơß den sich rasant ausbreitcnden Fernwirkungen von Arbeitsnicderlegungen kaum mehr durch einzelbetricbliche Maßnahmen vorgebeugt werden kann. ${ }^{363}$

\section{Erhöbung des gequerkschaftlicben Streikrisikos}

Vor diesem Hintergrund erscheint der vom BAG verfolgte Weg der Streikeindämmung allenfalls als Umweg, der jedoch nicht minder effektiv zum Ziel führt: Arbeitskämpfe können auch ohnc ihre autoritative Beschränkung dadurch verhindert werden, daß die Risiken, die für die kampfführende Partci mit ihnen verbunden sind, dcutlich erhöht werden. Muß sie damit rechnen, daß jede ihrer Akcionen mit legitimen Gegenmaßnahmen der anderen Seite beantwortet werden kann, so wird sie dies in ihr Kalkül aufnehmen und vermuclich behursamer zu Werk gehen, als wenn dies nicht der Fall wäre. Mí dieser Strategie der Risikomaximierung ergänzt das BAG die seit Beginn der $80 e r$ Jahre durch seine Rechesprechung zum Arbeitskampfrisiko"7 und durch die Änderung des $\$ 116$ AFG $^{\text {s }}$ veriolgte Politik einer Privatisierung der Arbeitskampfrisiken durch eine komplementäre Stcigenung der kollektivrechtichen Streikrisiken. Dem entspricht es, daß das Gericht in seinen neueren Entscheidungen weirgefaßiste Sorgfaltspflichten der Gewerkschaften im Hinblick auf Organisation, Durchführung und Überwachung von Streiks statuiert

\$6 Deutlich wurde dies kürelich an den Verhundlungen über concn neuen Manteltarifvertrag in der Druckunduxerse und fur das ZcıŁungsverlagsgew'crbe. Der Kündigung der bisherigen Mancelearifverträgc durch dic Gewerkschaft 7.um 31.12.198\$ hat der Bundesverband Druck dic Kündigung der Anhangc zum MTV emgegengesetzi. Der Arbeıtgeberverband verfolgt damı das Zie), dic dort enthateenen Besetzungsregeln an Maschinen und die Facharbesecrambindung zu revidicren. Die IG Druck rechncte: (zu Unreche) Pressebenchien zufolge in der Tarifauseinanderselzungen auch mit Angeiffsaussperrungen der Arbellgeber und warl ihnen vor, dic Verhandlungen von Beginn an nicht ernsthaft gefühn xu haben, vgl. den Benche in TAZ-Hamburg v. 2y-1.1959.

s6a Vgl, zu den mutmaßlichen Auswipkungen dieser Entwicklung de Beıtráge in: Bieback/Zechlin (Hrsg.),

Ende des Arberuskamples? Technikentwicklung und Sercik, Humburg $19 \$ 9$.

57 BAG, Beschlisse vom 22. $12.1980, \mathrm{BAG} \mathrm{E}_{z A} \mathrm{Nr}_{\mathrm{r}} 7$ und 8 xu $\$ 61, \mathrm{BGB}$, Betricbsrisiko.

s\& G v. Is. $5.19 \$ 6, B G B I 15.740$. 
und die juriscischen Zurechnungskriterien für ihre delikcischc Schadensersatzhaftung derart extensiv gefaßt hat, daß sie einer Gefährơungstraftung deurlich angenähert sind.

Danach isc eine Gcwerkschaft, die zum Streik aufruft, verpflichtet, "das Xampfverhalcen der Arbcitnehmer zu beobacliten und gegebenenfalls auf diesc einzuwirken, daß die Grenzen eines zulässigen Arbcitskampfes und cinzclner Arbeitskamplmaßnahmen nicht überschritten werden. "\$9 Da alle Maßnahmen unzulässig sind, die über bloßes Zureden, sich am Strcik zu beteiligen, hinausgehen ${ }^{60}$, haben die von der Gewerkschaft zu bestellenden Aufsichtsorgane dafür Sorge zu tragen, daß insbesondere die Behinderung arbeitswilliger Arbeitnehmer an Betrecen des Betriebes und die Verhindenng des $\mathrm{Zu}$ - und Abgangs von Waren und Kunden unterbleiben. Die örtlichen Streikleitungen sowvie dic sonscigen verantwortichen Funktionsträger dürfen solchc Handlungen nicht nur selbst nicht initiieren, an ihnen mitwirken oder sie billigend dulden, sondern sie haben gerade auch in emotional hochgradig angespannten Situationen die Pflicht, mäßigend auf die Streikenden einzuwirken ${ }^{6}$ Auch wenn die Gewerkschaft Blockadcaktionen weder veranlaßs noch von ihnen Kenntnis hat, so sind ihre Verterer gehalcen, auf entsprechende Hinweise der becroifenen Betriebe an den Ort des Geschehens zu cilen und zu versuchen, die rechtswidrigen Störungen zu bcenden. ${ }^{62}$ Tun sic dies nicht oder handeln die örtlichen Streikleiter als verfassungsmäßig berufene Organe der Gewerkschaít ihren Pflichten zuwider, so haftet die Gewerkschaft zwingend und in vollem Umfang für den dadurch dem Unternehmen entstandenen Schaden wegen rechtswidrigen Eingriffs in den eingerichteren und ausgeübten Gewerbebctrieb gem. $\$ 823$ Abs. ₹ i. Vb. mit $\$ 31$ BGB. Während sich die Gewerkschafe von der Haftung lür unerlaubue Handlungen von Streikposten nach $\$ 83$ BGB entasten kann, wenn sie nachweist, daß sie bei deren Auswahl und Unterweisung dic erforderliche Sorgfalt beachcer hat, ist sie für "Streikexzesse ihrer Organc ausnahmslos schadensersatzpflichtig - und dies, obwohl cin ansonsten rechtmäßiger A rocitskampl niche dadurch recheswidrig wird, "daß anläßlich diescs Arbciuskampfes streikende Arbeitnehmer, Streikposten oder Streikleitungen rechtswidrige Handlungen bcgehen, dic vom Streikrecht nicht gedeck sind ${ }^{6}{ }^{3}$ Der einzig verläßliche Weg, dieses weder nach Anlaß noch Umßang zu überblickende Haftungsrisiko selbst bei rechtmäßigen Streiks zu vermeiden, ist der Verzicht auf Arbeitskämpfe.

¿Das Mitcel des Streiks ist eine scharfe Waffe. Das verbieter es, das Streikrecht Pcrsonen oder Gruppen anzuvertrauen, bei denen nichc die Gewähr dafür besteht, daß sie nur in dem vercretbaren Umfang davon Gebrauch machen werden «. Mit diesem Argument hatte das BAG bercits in seiner ersten Entscheidung zum » wilden Streiks * vom 20.12.1963 $3^{64}$ begrindet, warum es nötig ist, "zu Kontrollzwecken Stellen einzuschalten, die wegen ihrer Stellung im Arbeitsleben, ihrer Bedeutung in wirtschaftlicher Hinsicht und ihrem Wissen auf dem Gebier des Arbeitskampfrechts dic Gewähr dafür bieten, daß nur in wirklich begründeten Fällen gestreikt wird und

59 Un. v. 21.6.1988 (Fn. 20), DB 1988. S. 1956.

6 Ebd., S. $195 s$.

61 Dies war spezsell in dem dec Entscheidung vom 21. 6. 1988, 2. a. O. (Fn. a 3) zugrundelicgenden Sachverhalı der Fall. Hier war con Bezurkssekretar der IG Druck und Papıer als örlicher Streiklester wahrend einer Sircik- und Blockadc(')akrion von cinem I.Icferantenfahrzeuc yweimai uherlahren worden, worauhin der anwesende 2. Landesvorsitzende der Gewerkschafe ausgerufen baben soll, hier sei cin Mord passiert, jetzi werde das Druckxentoum dicht gemacht. Das BAG weret ndic Verbrcilung der Morduhcser als schuldhalies Schüren von Emotionen, aufgrond dessen der Beklagic selbst soivic dic Gewerkschaft, falls die Behaupeungen der Klagerin auteclen, fur den ihr entstandenen Schaden hafien. 62 BAG. Urt. v. 8. 11.1988, a. a O. ([r. 23).

63 Urr. v. $21.5,1988,($ F n. 19), DB 1988, S. 1955.

64 A.2. O. (Fn, 8). 
daß im Falle eines Strciks dic im Allgemeininteresse erforderlichen Kampfregeln eingchatten werden ${ }^{65}$ Aus damaliger Siche kamen als solche verläßliche Kontrollstellen bei Strciks nur die Gewerkschaften in Fragc - mit der rechtsdogmacischen Konscquenz, daß die Kontrolleure zu den einzig legitimen Imbabern des Streikrechts und das Arbeitnehmergrundrecht des Art.9 Abs. 3 GG zum Verbandsgrundrecht umgestaltet wurde. ${ }^{66}$ Das BAG sieht sich heute offenbar vecanlaßt, die Gewerkscliaften nachdrücklich dàran zu crinnern, daß sie diese Kontrollaufgabe mic besonderer Intensitäı und Sorgfalc auszü̈bon haben.

Von der Steigerung dicser Sorgfalespfliche für - im Kern - eıgenes, vorausgegangenes gefährliches Handeln hin zum völligen Entzug der riskanten Handlungskompetenz ist logisch gesehen kein großer Schritr. Juristisch allerdings scehen Welten dazwischen, weil an ein generelles Streikverbou nicht (oder nur) zu denken ist. Der gleiche Effekt läßr sich jedoch auch dadurch erzielen, daß die gewerkschafıliche "Screikfreudigkeit " drastisch eingeschränkt wird.

\section{Und die Moral der Geschichte: Liberalisierung als Domestuzierung}

Die Logik der dargestellten Enzscheidungen entraltet sich in drei Schritten: Zunächst wird das Arbeitskampfrechr ganz und ausschließlich auf das System der Tarifautonomie hingeordnet. Sodann wird, im Bruch mit der Rechtsprechungsentwicklung der späten poer und der Soer Jahre, die differenzierte rechrliche Beurteilung und Gewichtung dor zulässigen Kampfmictel, dic zur Beschränkung der Zulässigkeit der Aussperrung und zur Privilegierung des gewerkschafelichen Warnstreiks geführt hatte, fallen gelassen und unter Preisgabe der ulcima-razio-Dokurina eine umfassende, lediglich durch das allfällige Verluältnismäßigkeitsprinzip beschränkte Arbcitskampffreiheit postuliert. Und schließlich, dics ist der krönende und das neuc Konstruke tragende Schlußscein, werden die Streikrisiken der Gewerkschaften und der Arbeitnchmer durch die Erweiterung ibrer delikrischen Schadensersaczhaftung in einer bislang ungeahnten Weise ausgedehnt, während dic ohnedics vergleichsweise bescheidenen Aussperrungsrisiken der Arbcitgeber minimicrt werden.

Die Entgrenzung der tarifbezogenen Arbeitskampffreiheit, dic auf den ersten Blick dem Märchenszenario einer (fast) konfliktireien Überflußgesellschaft kraß zu widersprechen scheint, cntpuppt ihren ticferen Sinn erst im Hinblick auf die Gewichte, mir denen diese Freiheit auf Arbeitnehmerseice bclaster wird: die Entgrenzung der legitimen Aussperrung und der Schadenshaftung für Streikexzessc. Ob diese neue Lastenvertcilung, die mit deutlicher Zeitverzögerung die reale Verschiebung der politisch-ökonomischen Einflußfakeoren auf das Macheverhälınis von Kapital und Arbeit seit Ende der joer Jahre rechtlich verdoppelt, den Effekt einer weiteren Reduktion der Strcikhäufigkeit und Domesrizierung der Strcikpraxis zur Folge haben wird, bleibc abzuwarten. Zumindest wird auf diese Weisc allen Ansäczen in den Gewerkschaften, aufgrund ihrer tawächlich oder nur vermeintlich sinkenden. Streiklähigkeit neve und effektivere Arbcitskampfformen auszuprobieren, cin Riegel vorgeschoben. ${ }^{67}$ Daran, daß dieser auf die Dauer auch tausächlich wirksam ist,

6) Ebd.

66 Unuberroffen poinuen ist noch ummer de Kruik von W/ethöler an der darun liegenden Verletzung der Koalitıonsfreihest der Arbeıtnehmer und der Umkehnung ihrer Schuizrechtc zu cencm gewerkschafilichen Anschlußzwong in seincm Funkkolleg, Rechiswissenschah. Frankfurd/M, $1968^{\circ}$ (unveranderitr Nachdruck Zunch 1986), S. 31 7 .

$67 \mathrm{Vgl}$. hıcrzu ewa dic Untersuchungen von Ostendor. Kriminalisicrung des Streikreches, Neuvud und 
besteht aufgrund der Erfahrungen insbesondere der britischen Gewerkschaften in den vergangenen Jahren kein $Z_{\text {wveifel }}{ }^{68}$ So soll ilinen, ob sie nun wollen oder niche, zunehmend stärker die Rollc eincs Kooperationspartners als eines Kontrahenden aufgenörigr werden mit der Perspekcive, das Märchen am Ende zur Wirklichkeir werden zu lassen und das Streikrechr gänzlich durch andere, aus Arbeitgebersicht gewiß sintelligentere" Alternativen zu ersetzen.

Doch wie enden Märchen? »Und wenn er nicht gestorben ist, dann lebt er heure noche.

Darmstade 1987 , von Zechlin, Strcik, Stralreche, Polizel, Köin 1986 und Lübbc-Wolff, Zur verfassungskonformen Inecrprciation des $\$ 823$ BGB um Zusammenliang mit akuv produktionsbehindernden AcbeiskampimaBnatimen, DB igR8 Beilage $9 / 1988$.

6s Vgl. Kaseendick, Zwischen Ausgre'nzung und kriscnpolieıscher Kondiuonıerung. Zur Situauon der bnuschen Gewcrkschaften, in: Muller-Jentsch (Hrsg.), Zukunft der Gewerkischalecr. Ein intcmatıonaler Vergleicl), FrankfurdM.-Ncw York, 19\$7, S, 160 ff. 